\title{
Photon and Jet Physics at the Collider Detector at Fermilab
}

\author{
J. R. Dittmann ${ }^{\text {a }}$ for the CDF collaboration
}

${ }^{a}$ Fermi National Accelerator Laboratory, M.S. 318, P.O. Box 500, Batavia, IL, 60510-0500, U.S.A.

We summarize recent Run 1 photon and jet measurements from $p \bar{p}$ collisions at $\sqrt{s}=0.63 \mathrm{TeV}$ and $1.8 \mathrm{TeV}$ using data collected at the Collider Detector at Fermilab (CDF). First Run 2 results at $\sqrt{s}=1.96 \mathrm{TeV}$ are also presented together with predictions of the kinematic reach accessible with $15 \mathrm{fb}^{-1}$ of Run 2 data. Data are compared to the predictions of Quantum Chromodynamics (QCD).

\section{Photon Physics at CDF}

The point-like coupling of photons to the hard interaction in high energy collisions offers a direct way to test the predictions of perturbative QCD. At lowest order, prompt photon production is dominated by the Compton process $g q \rightarrow \gamma q$, which is sensitive to the gluon distribution of the proton. The ratio of prompt photon cross sections measured at different center-of-mass energies provides a precise test of the QCD matrix elements, as many experimental uncertainties cancel and the theoretical uncertainties due to the gluon distribution are reduced.

A recent CDF measurement of the isolated prompt photon cross section at $\sqrt{s}=0.63$ and 1.8 TeV [1] reveals discrepancies between data and QCD that are difficult to explain with conventional theoretical uncertainties such as scale dependence and parton distribution parameterizations. Figure 1 highlights deviations from NLO QCD predictions [2] using the CTEQ5M parton distribution functions with a renormalization scale equal to the photon $P_{T}$. At low $P_{T}$, the data exhibit a steeper slope than theory, and at high $P_{T}$ the $\sqrt{s}=1.8 \mathrm{TeV}$ data and theory disagree by an overall normalization factor. The experimental results are reinforced by measurements of DØ [3] and UA2 [4]. One proposed explanation for the discrepancy at low $P_{T}$ is an inadequate description of the initial-state parton shower in the NLO QCD calculation which could give a recoil $k_{T}$ to the photon-jet system [5]. Including an additional $k_{T}$ recoil by adding simple Gaus-

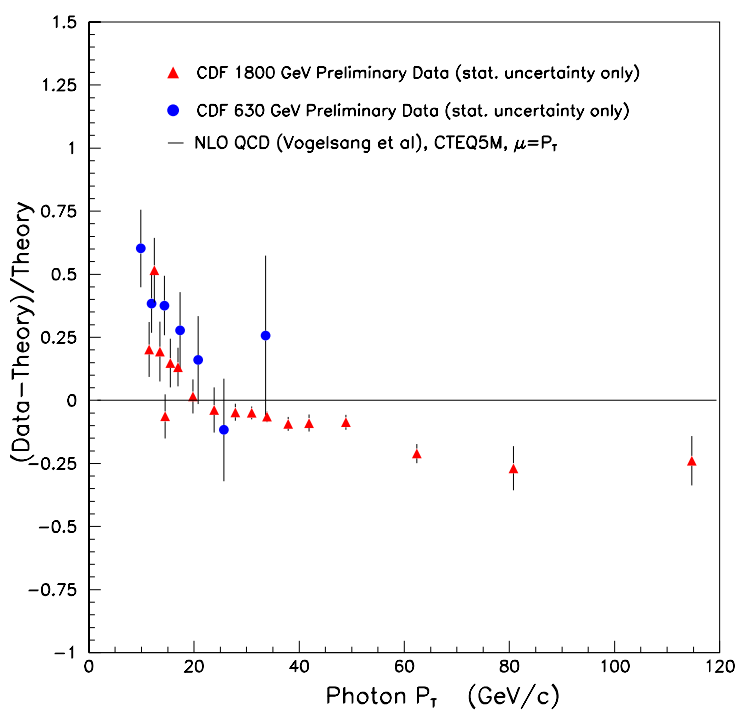

Figure 1. Comparison of the CDF isolated prompt photon cross section at $\sqrt{s}=0.63$ and 1.8 TeV to NLO QCD predictions.

sian smearing to the NLO QCD calculation yields better agreement with the data [1].

Studies of the prompt photon cross section will continue into Run 2 at CDF, where a full $15 \mathrm{fb}^{-1}$ of data are anticipated. In addition, other Run 2 photon analyses such as diphoton production and photon + heavy flavor production will play a leading role in tests of QCD and searches for new phenomena. In diphoton events, for example, where the final state kinematics can be completely reconstructed, the diphoton mass can be measured with good resolution out to nearly $600 \mathrm{GeV} / \mathrm{c}^{2}$. 


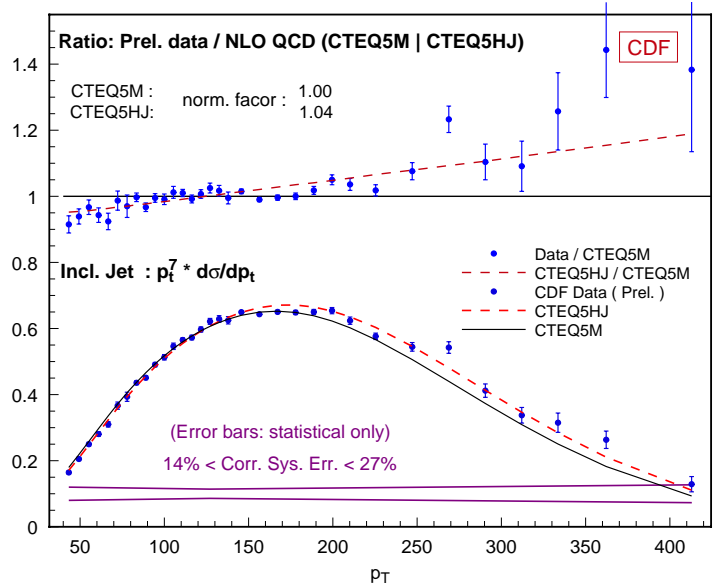

Figure 2. Ratio of the CDF inclusive jet cross section to NLO QCD theory using the CTEQ5M parton distribution functions. Predictions using CTEQ5HJ (dashed line) agree well with the data.

\section{Jet Physics at CDF}

\subsection{Inclusive jet cross section}

The inclusive jet cross section at CDF provides a fundamental test of QCD for jet energies ranging from 40-465 GeV, where the cross section changes by over 7 orders of magnitude. At the highest jet $E_{T}$, this measurement probes a distance scale of $\sim 10^{-17} \mathrm{~cm}$ and has traditionally been used to search for new physics.

Recent CDF Run 1B results [6] support the Run 1A observation of an excess in the jet cross section at large jet $E_{T}$ compared to NLO QCD predictions using parton distribution functions like MRST and CTEQ4M. Consistency between data and QCD can be attained, however, by virtue of a large uncertainty in the gluon distribution at high $x$. Figure 2 illustrates the level of agreement using CTEQ5HJ, where extra weight is given to high- $E_{T}$ jet data. Measurements of forward jets at $D \varnothing[7]$ further constrain the high$x$ gluon distribution, yielding a more "HJ-like" central fit for CTEQ6 that is consistent with both $\mathrm{CDF}$ and DØ inclusive jet data [8].

A large sample of Run 2 data will determine whether the high- $x$ gluon distribution completely explains the inclusive jet cross section at high $E_{T}$. For Run 2, the change in the center-of-mass energy from $\sqrt{s}=1.8$ to $1.96 \mathrm{TeV}$ increases the rate of high- $E_{T}$ jets by a factor of $2-3$, extending the reach beyond $650 \mathrm{GeV}$ in the central region. In addition, the new CDF endplug calorimeter will permit jet measurements in the forward region. These improvements, together with a suite of new jet algorithms, offer a rich program of study for jet physics in Run 2.

\subsection{Studies of the underlying event}

Precise jet measurements at hadron-hadron colliders require a good model of the "underlying event" which consists of multiple parton scattering, beam-beam remnants, and (part of) the initial and final-state radiation. Underlying event energy must be subtracted from jet energies in order to compare jet cross sections to NLO QCD. In addition, studies of the underlying event are worthwhile because they probe the interface between perturbative and non-perturbative QCD. Two complementary Run 1 analyses provide a quantitative study of the underlying event from very soft to very hard collisions.

In one analysis [9], charged particle tracks with $p_{T}>0.5 \mathrm{GeV} / \mathrm{c}$ and $|\eta|<1$ are identified in minimum bias and jet events. A simple algorithm is then applied to the list of tracks to locate "charged particle jets" with cone size $R=0.7$ in the $\eta-\phi$ plane. Tracks pointing to a region "transverse" to the direction of the highest- $p_{T}$ jet $\left(60^{\circ}\right.$ $<|\Delta \phi|<120^{\circ}$ ) are used to study the underlying event. Figure 3 illustrates the charged track multiplicity in the transverse region vs. the leading jet $p_{T}$, which extends up to $50 \mathrm{GeV} / \mathrm{c}$. Comparisons to PYтнIA 6.206 reveal that the default parameters underestimate the underlying event energy in the transverse region. The parameters that control the amount of initial-state radiation in Pyтнia can be tuned, however, to yield good agreement with data.

In the second analysis, jets are selected with $E_{T}$ ranging from $50-300 \mathrm{GeV}$. Charged tracks are observed in two cones at the same $\eta$ as the leading jet, but at $\pm 90^{\circ}$ away in $\phi$. Of the two cones, the one with the greater (lesser) $\Sigma p_{T}$ is defined as the $\max (\min )$ cone. The $p_{T}$ in the max cone increases with leading jet $E_{T}$, whereas the min cone $p_{T}$ stays constant at a level similar to that found in minimum bias events at $\sqrt{s}=1.8 \mathrm{TeV}$. 


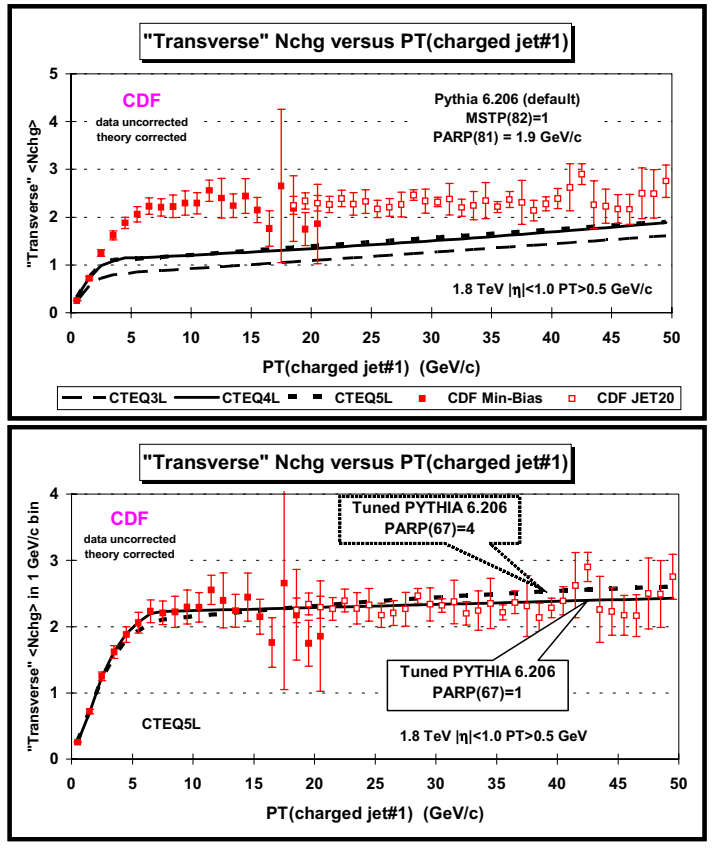

Figure 3. Mean number of charged tracks in the "transverse" region vs. the leading charged jet $p_{T}$ from Run 1 minimum bias and jet data. The data are compared to Pyтнia 6.206 using default parameters (top) and tuned parameters (bottom).

Although HERwIG agrees well with the data without any tuning, the parameters in PYTHIA that control the regularization scale for multiple parton scattering and the impact parameters option can be tuned to better fit the data.

\subsection{Three-jet production cross section}

In a new measurement of inclusive three-jet production at $\mathrm{CDF}$, features of three-jet events in $86 \mathrm{pb}^{-1}$ of Run 1B data are compared to NLO QCD predictions. Jets are identified using the CDF iterative cone algorithm (JETCLU) with cone size $R=0.7$ in the $\eta-\phi$ plane. Events are selected that have $\geq 3$ jets (each with $E_{T}^{\text {jet }}>20 \mathrm{GeV}$ and $\left.\left|\eta^{\text {et }}\right| \leq 2.0\right), \Sigma E_{T}(3$ jets $)>320 \mathrm{GeV}$, and a separation of $\Delta R>1.0$ between jets. These events are boosted into the three-jet rest frame, and the energies of the three leading jets are corrected, unsmeared, and numbered such that $E_{3}>E_{4}>E_{5}$. The three-jet mass $m_{3 \text { jet }}$ is calculated together with the Dalitz variables $X_{i}=2 E_{i} / m_{3 \mathrm{jet}}$.

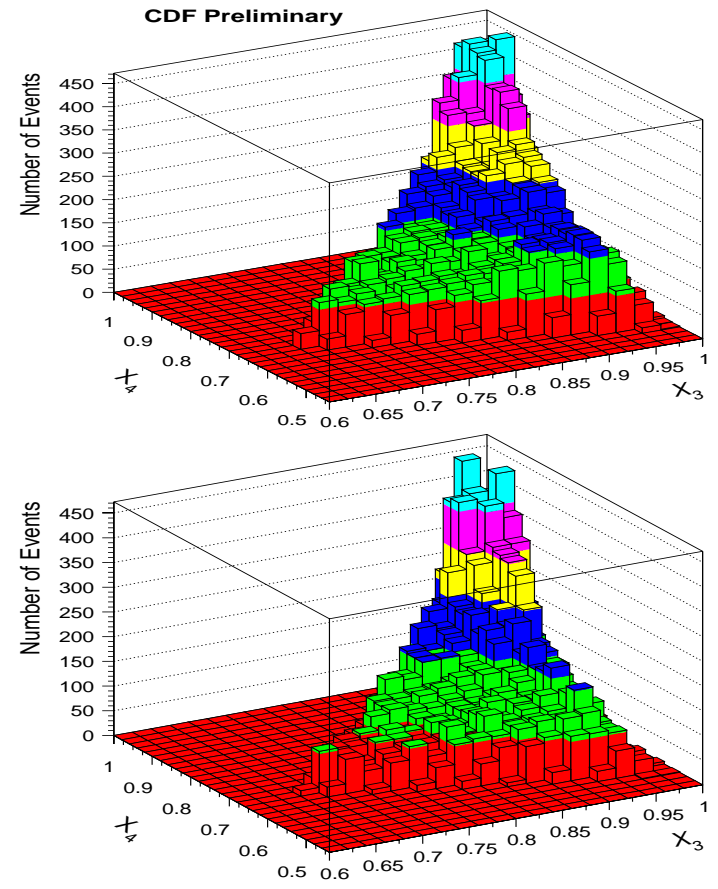

Figure 4. Event density in the $X_{3}-X_{4}$ plane for three-jet events in Run 1B data (top) and NLO QCD (bottom). The theoretical cross section is calculated using CTEQ3 with $\alpha_{s}=0.116$.

Figure 4 compares the three-jet cross section vs. $X_{3}$ and $X_{4}$ to NLO QCD predictions [10]. A detailed, bin-by-bin comparison of data and theory indicates good agreement. The total measured three-jet production cross section, using the full kinematically-allowed Dalitz plane, is $466 \pm$ 2 (stat) ${ }_{-71}^{+206}$ (syst) pb, which is consistent with the NLO QCD prediction of $402 \pm 3 \mathrm{pb}$.

\subsection{Jet shapes in inclusive dijet events}

The internal structure of jets is mainly dictated by multi-gluon emission from the primary parton which involves higher-order QCD processes. The study of this structure thus provides a stringent test of the models used to simulate parton showers. Here we present preliminary jet shape measurements using a sample of inclusive dijet events in $16 \mathrm{pb}^{-1}$ of Run 2 CDF data.

Jets are identified using JETCLU with cone size $R=0.7$. Events are selected that contain at least two jets with $E_{T}^{\text {jet }}>30 \mathrm{GeV}$ and $\left|\eta^{\text {jet }}\right|<2.3$. 


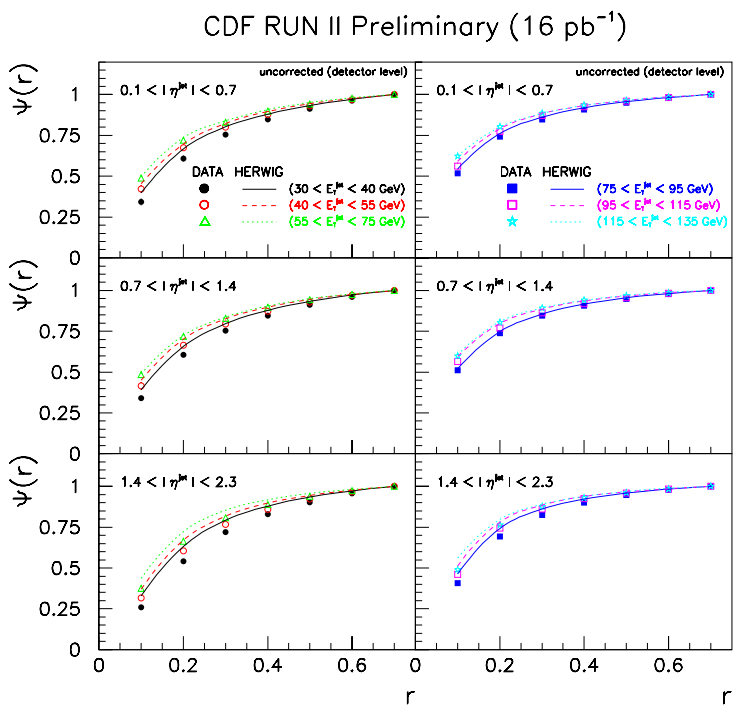

Figure 5. Integrated jet shapes, $\Psi(r)$, for various bins of $E_{T}^{\text {jet }}$ and $\eta^{\text {jet }}$, measured using the calorimeter. The data are compared to Herwig Monte Carlo predictions.

The integrated jet shape, $\Psi(r)$, is defined as the average fraction of the jet's transverse energy that lies inside an inner cone of radius $r$ concentric to the jet cone:

$\Psi(r)=\frac{1}{N_{\text {jet }}} \Sigma_{\text {jets }} \frac{E_{T}(0, r)}{E_{T}(0, R)} \quad(r<R)$

A plot of $\Psi(r)$ for jets with $30 \mathrm{GeV}<E_{T}^{\text {jet }}<$ $135 \mathrm{GeV}$ and $0.1<\left|\eta^{\text {jet }}\right|<0.7$ is shown in Figure 5. Jets become narrower as $E_{T}^{\text {jet }}$ increases, but no significant dependence on $\eta^{\text {jet }}$ is observed. The data are compared to HERWIG predictions with initial and final-state radiation and interactions between spectator partons ("underlying event"). Data and theory agree very well, although HERWIG predicts jets that are too narrow at low $E_{T}^{\text {jet }}$ and high $\eta^{\text {jet }}$. This disagreement may be due to the modeling of the underlying event.

The CDF Central Outer Tracker (COT) provides an independent method to reconstruct the jet shape, thereby validating the calorimeterbased measurement. For a given jet, tracks that point within the jet cone are identified and $\Psi(r)$ is calculated based on the $p_{T}$ of the tracks simi-

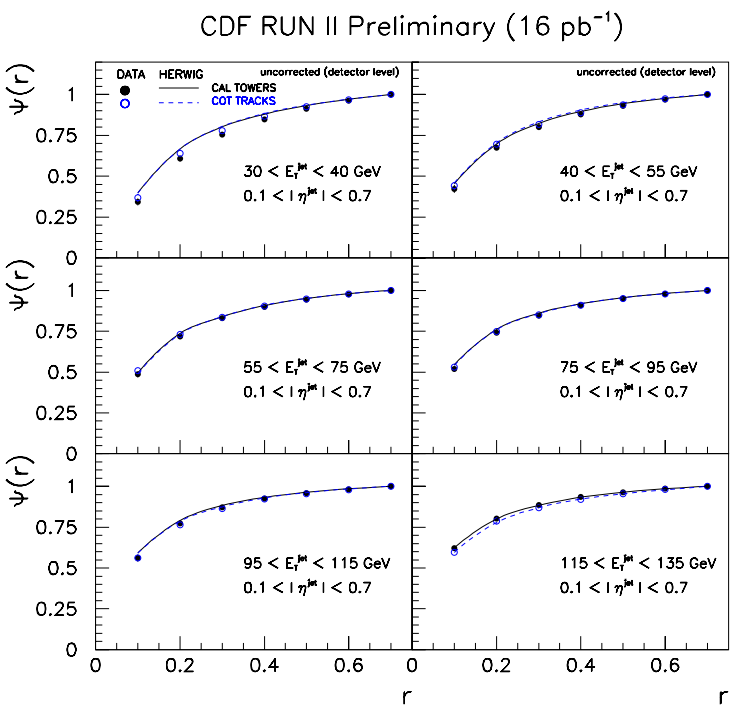

Figure 6. Measured integrated jet shapes, $\Psi(r)$, using calorimeter towers (solid circles) or COT tracks (open circles).

lar to Eq. 1. Figure 6 compares the integrated jet shapes for calorimeter towers and tracks in a region with good COT coverage $\left(0.1<\left|\eta^{\text {jet }}\right|<0.7\right)$. Excellent agreement between the calorimeter and tracking measurements is observed.

\section{REFERENCES}

1. D. Acosta et al., Phys. Rev. D 65, 112003 (2002).

2. M. Gluck et al., Phys. Rev. Lett. 73, 388 (1994).

3. B. Abbott et al., Phys. Rev. Lett. 84, 2786 (2000).

4. J. Alitti et al., Phys. Lett. B 263, 544 (1991).

5. L. Apanasevich et al., Phys. Rev. D 59, 074007 (1999).

6. T. Affolder et al., Phys. Rev. D 64, 032001 (2001).

7. B. Abbott et al., Phys. Rev. Lett. 86, 1707 (2001).

8. M. Zielinski, these proceedings.

9. T. Affolder et al., Phys. Rev. D 65, 092002 (2002).

10. W. Kilgore and W. Giele, hep-ph/0009193. 